\title{
Computing Recomposition of Maps with a New Sampling Asymptotic Formula
}

\author{
Almudena Antuña ${ }^{1}$, Juan L. G. Guirao², Miguel A. López ${ }^{3}$ \\ ${ }^{1}$ Departamento de Análisis Económico y Finanzas, Universidad de Castilla-La Mancha, \\ Cuenca (Castilla-La Mancha), Spain \\ ${ }^{2}$ Departamento de Matemática Aplicada y Estadstica, Universidad Politécnica de Cartagena, \\ Cartagena (Región de Murcia), Spain \\ ${ }^{3}$ Departamento de Matemáticas, Universidad de Castilla-La Mancha, \\ Cuenca (Castilla-La Mancha), Spain \\ E-mail: almudena.antuna@uclm.es,juan.garcia@upct.es,mangel.lopez@uclm.es \\ Received April 6, 2011, revised May 5, 2011, accepted May 21, 2011
}

\begin{abstract}
The aim of the present paper is to state an asymptotic property $\mathcal{P}$ of Shannon's sampling theorem type, based on normalized cardinal sines, and keeping constant the sampling frequency of a not necessarilly bandlimited signal. It generalizes in the limit the results stated by Marvasti et al. [7] and Agud et al. [1]. We show that $\mathcal{P}$ is fulfilled for any constant signal working for every given sampling frequency. Moreover, we conjecture that Gaussian maps of the form $\mathrm{e}^{-\lambda t^{2}}, \lambda \in \mathbb{R}^{+}$, hold $\mathcal{P}$. We support this conjecture by proving the equality given by $\mathcal{P}$ for the three first coefficients of the power series representation of $\mathrm{e}^{-\lambda t^{2}}$.
\end{abstract}

Keywords: Band-Limited Signal, Shannon's Sampling Theorem, Approximation Theory

\section{Introduction and Statement of the Main Results}

A central result of the signal theory in engineering is the well-known Shannon-Whittaker-Kotel'nikov's theorem (see for instance [9] or [11]) working for band-limited maps of $L^{2}(\mathbb{R})$ (i.e., for Paley-Wiener signals), and based on the normalized cardinal sinus map $\operatorname{sinc}(t)$ defined by

$$
\operatorname{sinc}(t)=\left\{\begin{array}{cc}
1 & \text { if } t=0, \\
\frac{\sin (\pi t)}{\pi t} & \text { if } t \neq 0 .
\end{array}\right.
$$

Another philosopher's stone of the signal processing theory is the Middleton's sampling theorem for band step functions (see [8]). This result was one of the first modifications of the classic Sampling theorem (see [10]) which only works for band-limited maps. After this starting point many different extensions and generalizations of this theorem appeared in the literature trying to

*This work has been partially supported by MCI (Ministerio de Ciencia e Innovación) and FEDER (Fondo Europeo Desarrollo Regional), grant number MTM2008--03679/MTM, Fundación Séneca de la Región de Murcia, grant number 08667/PI/08 and JCCM (Junta de Comunidades de Castilla-La Mancha), grant number PEII09-0220-0222. obtain approximations of non band-limited signals (see for instance [2] or [4]). Good surveys on these extensions are [3] or [11].

In this paper we follow the spirit of the previous results in the sense of trying to obtain approximations of non band-limited signals by using band-limited ones by increasing the band size. But our approach is completely different to the previous ones in the sense that we keep constant the sampling frequency generalizing in the limit the results of Marvasti et al. [7] and Agud et al. [1] .

In this setting, we state the following asymptotic property of Shannon's sampling theorem type where the convergence is considered in the Cauchy's principal value for the series and pointwise for the limit.

Property 1 Let $f: \mathbb{R} \rightarrow \mathbb{R}$ be a map and $\tau \in \mathbb{R}^{+}$. We say that $f$ holds the property $\mathcal{P}$ for $\tau$ if

$$
f(t)=\lim _{n \rightarrow \infty}\left(\sum_{k \in \mathbb{Z}} f^{\frac{1}{n}}\left(\frac{k}{\tau}\right) \operatorname{sinc}(\tau t-k)\right)^{n} .
$$

The statement of the main results is:

Theorem 1 Every constant signal holds property $\mathcal{P}$ for every given $\tau \in \mathbb{R}^{+}$.

Conjecture 1 The Gaussian maps, i.e. maps of the form $\mathrm{e}^{-\lambda t^{2}}, \lambda \in \mathbb{R}^{+}$hold property $\mathcal{P}$ for every given 
$\tau \in \mathbb{R}^{+}$.

To support our feeling on the truth of the Conjecture 1 we prove, without loss of generality for $\lambda=1$, that the Gaussian map $\mathrm{e}^{-t^{2}}$ holds expression (1) for the three first coefficients of the power series representation of $\mathrm{e}^{-t^{2}}$. Note that since the Gaussian map is analytical, for proving formula (1) is enough to show the equality between the coefficients of the power series representation of the Gaussian map and the coefficients of the series stated in the second member of (1) after proving the analitycity of the second member of (1). The statement of our result is the following:

Theorem 2 Let $\mathrm{e}^{-t^{2}}$ be a Gaussian map. Then the three first coefficients of the power series representation of $\mathrm{e}^{-t^{2}}$ are equal to the three first ones of the second member of expression (1).

The paper is divided into three sections. In Section 2 we present the ideas and results that have inspired us to formulate property $\mathcal{P}$ and Conjecture 1 . Section 3 is devoted to prove Theorem 1 and in Section 4 is proved Theorem 2 .

\section{On the Property $\mathcal{P}$ and Conjecture 1}

We state as a property $\mathcal{P}$ an approximation in the limit, through potentials of band--limited maps of the original signal, based on [1] and [7].

In [1] is proven that given a sequence $\left\{s_{k}\right\}_{k \in \mathbb{Z}} \in l^{2 / n}(\mathbb{Z})$, $B>0, \tau \geq 2 B$ and $n$ odd, there exist exactly $n$ band-limited signals $\left\{x_{r}\right\}$ with bandwidth equal to $B$ such that $x_{r}^{n}\left(\frac{k}{\tau}\right)=s_{k}$. Moreover, is shown that

$x_{r}=e_{r} x_{0}$, where $\left\{e_{r}\right\}_{r=0}^{n-1}$ are the roots of unity of order $n$ and $x_{0}(t)=\sum_{k \in Z} s_{k}^{1 / n} \operatorname{sinc}(2 B t-k)$.

From this is directly deduced that if we consider an odd number $n$ and a band-limited signal $f$ with bandwidth $\tilde{B}$ such that the sequence of coefficients

$\left\{f\left(\frac{k}{\tau}\right) ; k \in \mathbb{Z}\right\}$ with $\tau \geq \frac{2 \tilde{B}}{n}$ holds the properties stated in [1], then the signal admits a recomposition of Shannon type in the form

$$
f(t)=\left(\sum_{k \in \mathbb{Z}} f^{\frac{1}{n}}\left(\frac{k}{\tau}\right) \operatorname{sinc}(\tau t-k)\right)^{n},
$$

where clearly the sampling frequency can be choosen bigger than the Nyquist one.

Our aim is to provide a method for approximating non band-limited signal by band-limited ones and keeping the frequency of the sampling constant. And our idea is to take limits in (2) obtaining an equality of the form

$$
f(t)=\lim _{n \rightarrow \infty}\left(\sum_{k \in \infty} f^{\frac{1}{n}}\left(\frac{k}{\tau}\right) \operatorname{sinc}(\tau t-k)\right)^{n},
$$

expressed as a property $\mathcal{P}$.

In Section 3 we prove that property $\mathcal{P}$ is held by any constant map for every $\tau \in \mathbb{R}^{+}$. Thus, the universe of non-trivial signals which hold the conjecture is nonempty (note that $f(t) \equiv 0$ holds $\mathcal{P}$ ). Our feeling is that there are a big number of representative signals in engineering processes which satisfy property $\mathcal{P}$.

We state as Conjecture 1 to prove that any signal of Gaussian type holds the statement. Note that the Gaussian map, which is mathematically important in itself, plays an important role in the signal theory because the Gaussian map is the unique function which reachs the minimum of the product of the temporal and frecuential width. This minimum is given by the Uncertainty Principle, see [6]. We believe in the working of Conjecture 1 and we support it through Theorem 2 where we show the equality between the three first coefficients of the power series representation of the Gaussian map and property $\mathcal{P}$. For proving completely the conjecture, by the analyticity of the Gaussian map, is enough to prove that expression

$\lim _{n \rightarrow \infty}\left(\sum_{k \in \mathbb{Z}} \mathrm{e}^{-\frac{k^{2}}{n \tau^{2}}} \operatorname{sinc}(\tau t-k)\right)^{n}$ defines an analytical map and to show that the equality works for the rest of coefficients.

\section{Proof of Theorem 1}

The following lemma will play a key role in the proof of Theorem 1.

Lemma $3 \sum_{k \in \mathbb{Z}} \operatorname{sinc}(z-k)=1$ for every $z \in \mathbb{C}$.

Proof. First of all we shall show that the result works for every $t \in \mathbb{R}$. Indeed, if $t \in \mathbb{Z}$, the result is straight because of

$$
\sum_{k \in \mathbb{Z}} \operatorname{sinc}(t-k)=1+\sum_{\substack{k \in \mathbb{Z} \\ k \neq t}} \operatorname{sinc}(t-k)=1+0=1 .
$$

Therefore, from now on we assume that $t \in \mathbb{R} \backslash \mathbb{Z}$. Taking simetric terms in the series we obtain

$$
\begin{aligned}
& \sum_{k \in \mathbb{Z}} \operatorname{sinc}(t-k)=\frac{\sin (\pi t)}{\pi t} \\
& +\sum_{k \in \mathbb{N}}\left(\frac{\sin (\pi(t-k))}{\pi(t-k)}+\frac{\sin (\pi(t+k))}{\pi(t+k)}\right) \\
& =\frac{\sin (\pi t)}{\pi t}+\frac{2 t \sin (\pi t)}{\pi} \sum_{k \in \mathbb{N}} \frac{(-1)^{k+1}}{k^{2}-t^{2}}
\end{aligned}
$$

On the other hand, for a given $t \in \mathbb{R} \backslash \mathbb{Z}$ is known 
that

$$
\frac{t \pi}{\sin (t \pi)}=1+2 t^{2} \sum_{k \in \mathbb{N}} \frac{(-1)^{k}}{t^{2}-k^{2}}
$$

and therefore

$$
\sum_{k \in \mathbb{N}} \frac{(-1)^{k+1}}{k^{2}-t^{2}}=\frac{-1}{2 t^{2}}+\frac{\pi}{2 t \sin (\pi t)}
$$

Finally, replacing (4) in expression (3) the proof is over for every real number $t$.

The prove of the result for complex numbers is a consequence of the use of the Analytic Prologation Principle. For applying it, is enough to prove that the series $\sum_{k \in \mathbb{Z}} \operatorname{sinc}(z-k)$ is an analytic function. Indeed, by (3) the series can be written in the form

$$
\sum_{k \in \mathbb{Z}} \operatorname{sinc}(z-k)=\frac{\sin (\pi z)}{\pi z}+\frac{2 z \sin (\pi z)}{\pi} \sum_{k \in \mathbb{Z}} \frac{(-1)^{k+1}}{k^{2}-z^{2}} .
$$

Obviously, the first term of the previous sum is an analytic map. For proving the analyticity of the second term of the sum we shall prove that the series

$\sum_{k \in \mathbb{N}} \frac{(-1)^{k+1}}{k^{2}-z^{2}}$ uniformly converges on every compact set $L \subset \mathbb{C} \backslash \mathbb{N}$. In fact, let $s=\max \{|z|: z \in L\}$ and $k_{0}$ be such that $k_{0}>2 s$, then for every $k \geq k_{0}$ is $|z|<\frac{k}{2}$ for every $z \in L$. Therefore,

$$
\left|\frac{(-1)^{k+1}}{k^{2}-z^{2}}\right| \leq \frac{4}{3 k^{2}}
$$

which guarantees the uniformly convergency of the series in $L$ and the proof is over.

Remark 4 We underline that the fact of the series $\sum_{k \in \mathbb{Z}} \operatorname{sinc}(z-k)$ defines an analytic function is a direct consequence of the application of the Uniform Convergence Principle for cardinal Series, see [5, pag. 70] or [11, pag. 22] for a more up-to-date reference. We present a direct approach in the proof of Lemma 3 for completness of the arguments.

Proof of Theorem 1. Let $f(t)=C$ be a constant signal and $\tau \in \mathbb{R}^{+}$. By Lemma 3 we have

$$
\begin{aligned}
& \lim _{n \rightarrow \infty}\left(\sum_{k \in \mathbb{Z}} f^{\frac{1}{n}}\left(\frac{k}{\tau}\right) \operatorname{sinc}(t \tau-k)\right)^{n} \\
& =\lim _{n \rightarrow \infty}\left(C^{\frac{1}{n}} \sum_{k \in \mathbb{Z}} \operatorname{sinc}(t \tau-k)\right)^{n} \\
& =\lim _{n \rightarrow \infty} C\left(\sum_{k \in \mathbb{Z}} \operatorname{sinc}(t \tau-k)\right)^{n}=\lim _{n \rightarrow \infty} C=C .
\end{aligned}
$$

Thus, is shown that $f$ holds property $\mathcal{P}$ ending the proof.

\section{Proof of Theorem 2}

In the sequel we denote by $J$ a set of consecutive natural numbers in the form $\{0,1,2, \cdots\}$ which eventually can be $\mathbb{N} \cup\{0\}$. By $\#(J)$ we denote the cardinal of the set $J$ and we assume the arithmetic of the infinity (i.e., $\forall k \in \mathbb{N}, \infty \pm k=\infty$ ), therefore by $m_{J}$ we denote $\#(J)-1$.

Given a sequence $\alpha=\left\{\alpha_{n}\right\}_{n \in J}$ of real numbers, by $d(\alpha)$ we denote the diameter of the sequence $\alpha$, i.e., $d(\alpha)=\sup _{1 \leq n<\#(J)}\left\{\left|\alpha_{n}-\alpha_{n-1}\right|\right\}$. As usual by [.] we denote the integer part.

Lemma 5 Let $\gamma=\left\{\gamma_{n}\right\}_{n \in J}$ be an increasing bounded sequence of real numbers holding the following conditions:

1) $a=\gamma_{0}<\gamma_{1}<\cdots<\gamma_{n-1}<\gamma_{n}<\cdots<b=\sup _{n \in J}\left\{\gamma_{n}\right\}$,

2) $\left\{\gamma_{n}-\gamma_{n-1}\right\}_{n \in J \backslash\{0\}}$ is monotonic.

Let $f:[a, b] \rightarrow \mathbb{R}$ be a continuous map of constant sign on $[a, b]$, eventually $f$ can be equal to zero. Then for every sequence $\beta=\left\{\beta_{n}\right\}_{n \in J \backslash\{0\}}$ such that $\beta_{k} \in\left[\gamma_{k-1}, \gamma_{k}\right]$ and for every $\varepsilon>0$ there exists $\delta>0$ such that if $d(\gamma)<\delta$ then

$$
\left|\sum_{k=1}^{M} f\left(\beta_{2 k}\right)\left(\gamma_{2 k}-\gamma_{2 k-1}\right)-\frac{1}{2} \int_{a}^{b} f(x) \mathrm{d} x\right|<\varepsilon
$$

and

$$
\left|\sum_{k=0}^{L} f\left(\beta_{2 k+1}\right)\left(\gamma_{2 k+1}-\gamma_{2 k}\right)-\frac{1}{2} \int_{a}^{b} f(x) \mathrm{d} x\right|<\varepsilon,
$$

where $M=\left[\frac{m_{J}}{2}\right]$ and $L=\left[\frac{m_{J}-1}{2}\right]$.

Proof. For proving (5) we assume, without loss of generality, that $f \geq 0$ and $\left\{\gamma_{n}-\gamma_{n-1}\right\}_{n \in J \backslash\{0\}}$ is a decreasing sequence. We shall use the following notation

$$
\begin{gathered}
T_{o}(\boldsymbol{\gamma}, \boldsymbol{\beta})=\sum_{k=0}^{L} f\left(\beta_{2 k+1}\right)\left(\gamma_{2 k+1}-\gamma_{2 k}\right), \\
T_{e}(\boldsymbol{\gamma}, \boldsymbol{\beta})=\sum_{k=1}^{M} f\left(\beta_{2 k}\right)\left(\gamma_{2 k}-\gamma_{2 k-1}\right), \\
S_{l e}(\boldsymbol{\gamma})=\sum_{k=1}^{M} f\left(\gamma_{2 k}\right)\left(\gamma_{2 k}-\gamma_{2 k-1}\right), \\
S_{r e}(\boldsymbol{\gamma})=\sum_{k=0}^{L} f\left(\gamma_{2 k}\right)\left(\gamma_{2 k+1}-\gamma_{2 k}\right), \\
S_{r o}(\boldsymbol{\gamma})=\sum_{k=1}^{M} f\left(\gamma_{2 k-1}\right)\left(\gamma_{2 k}-\gamma_{2 k-1}\right),
\end{gathered}
$$




$$
S_{l o}(\gamma)=\sum_{k=0}^{L} f\left(\gamma_{2 k+1}\right)\left(\gamma_{2 k+1}-\gamma_{2 k}\right) .
$$

For a given $\varepsilon>0$, since $T_{o}+T_{e}$ is a Riemann sum of $f$ on $[a, b]$, there exists $\delta_{0}>0$ such that if $d(\gamma)<\delta_{0}$, then

$$
\left|T_{o}+T_{e}-\int_{a}^{b} f(x) \mathrm{d} x\right|<\varepsilon .
$$

Taking $\varepsilon_{1}=\frac{\varepsilon}{3(b-a)}>0$, since the map $f$ is uniformly continuous on the interval $[a, b]$, then there exists $\delta_{1}>0$ such that if $\left|\beta_{2 k}-\gamma_{2 k}\right|<\delta_{1}$ then $\left|f\left(\beta_{2 k}\right)-f\left(\gamma_{2 k}\right)\right|<\varepsilon_{1}$ and consequently if $d(\gamma)<\delta_{1}$

$$
\begin{aligned}
\left|T_{e}-S_{l e}\right| & =\left|\sum_{k=1}^{M}\left(f\left(\beta_{2 k}\right)-f\left(\gamma_{2 k}\right)\right)\left(\gamma_{2 k}-\gamma_{2 k-1}\right)\right| \\
& <\varepsilon_{1} \sum_{k=1}^{M}\left|\gamma_{2 k}-\gamma_{2 k-1}\right|<\varepsilon_{1}(b-a)=\frac{\varepsilon}{3} .
\end{aligned}
$$

Proceeding in a similar way

$$
\left|T_{o}-S_{r e}\right|<\frac{\varepsilon}{3},\left|T_{e}-S_{r o}\right|<\frac{\varepsilon}{3} \text { and }\left|T_{o}-S_{l o}\right|<\frac{\varepsilon}{3} .
$$

Now, it is easily deduced that

$$
f\left(\gamma_{2 k}\right)\left(\gamma_{2 k+1}-\gamma_{2 k}\right) \leq f\left(\gamma_{2 k}\right)\left(\gamma_{2 k}-\gamma_{2 k-1}\right),
$$

and

$$
S_{r e}-S_{l e} \leq f\left(\gamma_{0}\right)\left(\gamma_{1}-\gamma_{0}\right) \text {. }
$$

So, taking $\delta_{2}=\min \left\{\delta_{1}, \frac{\varepsilon}{3 f\left(\gamma_{0}\right)}\right\}$ if $f\left(\gamma_{0}\right) \neq 0$ and $\delta_{2}=\delta_{1}$ in other case, if $d(\gamma)<\delta_{2}$ then

$$
S_{r e}-S_{l e}<f\left(\gamma_{0}\right) \delta_{2}<\frac{\varepsilon}{3} \text {. }
$$

Using the previous inequality, () and () we have that

$$
T_{o}-T_{e}=\left(T_{o}-S_{r e}\right)+\left(S_{l e}-T_{e}\right)+\left(S_{r e}-S_{l e}\right)<\varepsilon .
$$

On the other hand, it is clear that

$$
S_{r o}-S_{l o} \leq 0
$$

and so, using (9) and (11)

$$
T_{e}-T_{o}=\left(T_{e}-S_{r o}\right)+\left(S_{l o}-T_{o}\right)+\left(S_{r o}-S_{l o}\right)<\frac{2 \varepsilon}{3}<\varepsilon .
$$

From here and $(10)$, if $d(\gamma)<\delta_{2}$,

$$
\left|T_{e}-T_{0}\right|<\varepsilon \text {. }
$$

So, taking $\delta=\min \left\{\delta_{0}, \delta_{2}\right\}$ and using the previous inequality and (7), if $d(\gamma)<\delta$ then

$$
\begin{aligned}
& \left|T_{e}(\gamma)-\frac{1}{2} \int_{a}^{b} f(x) \mathrm{d} x\right| \leq \frac{1}{2}\left|T_{e}(\gamma)-T_{o}(\gamma)\right| \\
& +\frac{1}{2}\left|T_{e}(\gamma)+T_{o}(\gamma)-\int_{a}^{b} f(x) \mathrm{d} x\right|<\varepsilon,
\end{aligned}
$$

which is just (5) as we want to show.

The proof of (6) follows in an analogous way.

Lemma 6 Let $x \in \mathbb{R}^{+}, k \in \mathbb{N}$ and

$l_{k}(x)=\frac{1-\mathrm{e}^{-k^{2} x}}{k^{2} x}$. Then for every $k$ is

$\lim _{x \rightarrow 0^{+}}\left(l_{k}(x)-l_{k+1}(x)\right)=0$ uniformly in $k$.

Proof. Note that for every $x \in \mathbb{R}^{+}$and every $k \in \mathbb{N}$, $l_{k}(x)$ is decreasing in $k$.

We fixed $x \in(0,1)$. For a given $\varepsilon>0$ there exist $C>0$ holding $l_{k}(x)<\frac{\varepsilon}{2}$ for any $k$ such that $k^{2} x \geq C$ and consequently

$$
l_{k}(x)-l_{k+1}(x)<\varepsilon .
$$

On the other hand, using the power series representation of the exponential function and the Newton's binomial,

$$
\begin{aligned}
& l_{k}(x)-l_{k+1}(x) \\
& =\sum_{p=0}^{\infty} \frac{(-1)^{p}}{(p+1) !}\left(\left(k^{2} x\right)^{p}-\left((k+1)^{2} x\right)^{p}\right) \\
& =\sqrt{x} \sum_{p=0}^{\infty} \frac{(-1)^{p+1}}{(p+1) !} \sum_{q=0}^{2 p-1}\left(\begin{array}{c}
2 p \\
q
\end{array}\right)(k \sqrt{x})^{q} x^{p-\frac{q+1}{2}} \\
& \leq \sqrt{x} \sum_{p=0}^{\infty} \frac{1}{(p+1) !}(1+k \sqrt{x})^{2 p} \\
& =\sqrt{x} \mathrm{e} \frac{(1+k \sqrt{x})^{2}-1}{(1+k \sqrt{x})^{2}}
\end{aligned}
$$

and if $k^{2} x<C$ we have the following inequality

$$
l_{k}(x)-l_{k+1}(x)<\sqrt{x}\left(\mathrm{e}^{(1+\sqrt{C})^{2}}-1\right) .
$$

Since $\lim _{x \rightarrow 0} \sqrt{x}\left(\mathrm{e}^{(1+\sqrt{C})^{2}}-1\right)=0$, using the last inequality and (12) the proof is over.

The following proposition will play a key role in the proof of Theorem 2.

Proposition 7 Let $x \in \mathbb{R}^{+}$and $L(x)=\sum_{k \in \mathbb{N}}(-1)^{k+1} l_{k}(x)$. Then is held

$$
L(x) \leq \frac{\pi}{2} \text { and } \lim _{x \rightarrow 0^{+}} L(x)=\frac{1}{2} .
$$

Proof. We consider the functions $\alpha_{k}(x)=\operatorname{arctgl}_{k}(x)$ 
on $\left[0, \frac{\pi}{2}\right]$. Let $x \in \mathbb{R}^{+}$fixed. We note that $\alpha_{k}(x)$ is a decreasing sequence on $k$. It is easily deduced that using the Intermediate Value Theorem

$$
\begin{aligned}
L(x) & =\sum_{k \in \mathbb{N}}\left(l_{2 k-1}(x)-l_{2 k}(x)\right) \\
& =\sum_{k \in \mathbb{N}}\left(\operatorname{tg} \alpha_{2 k-1}(x)-\operatorname{tg} \alpha_{2 k}(x)\right),
\end{aligned}
$$

and therefore

$$
L(x)=\sum_{k \in \mathbb{N}} \frac{\alpha_{2 k-1}(x)-\alpha_{2 k}(x)}{\cos ^{2} \beta_{2 k-1}},
$$

for suitable $\beta_{2 k-1} \in\left(\alpha_{2 k}(x), \alpha_{2 k-1}(x)\right)$.

Note that $\alpha_{k}(x) \in\left(0, \frac{\pi}{4}\right)$ for all $k \in \mathbb{N}$ and consequently $0<\beta_{k}<\frac{\pi}{4}$. Thus,

$$
\begin{aligned}
L(x) & \leq 2 \sum_{k \in \mathbb{N}}\left(\alpha_{2 k-1}(x)-\alpha_{2 k}(x)\right) \\
& \leq 2 \sum_{k \in \mathbb{N}}\left(\alpha_{k}(x)-\alpha_{k+1}(x)\right)=2 \alpha_{1}(x) \leq \frac{\pi}{2} .
\end{aligned}
$$

Since $\int_{0}^{\frac{\pi}{4}} \frac{\mathrm{d} t}{\cos ^{2} t}=1$, then

$$
\begin{aligned}
& \left|L(x)-\frac{1}{2}\right|=\left|L(x)-\frac{1}{2} \int_{0}^{\frac{\pi}{4}} \frac{\mathrm{d} t}{\cos ^{2} t}\right| \\
& \leq\left|L(x)-\frac{1}{2} \int_{0}^{\alpha_{1}(x)} \frac{\mathrm{d} t}{\cos ^{2}(t)}\right|+\left|\frac{1}{2} \int_{\alpha_{1}(x)}^{\frac{\pi}{4}} \frac{\mathrm{d} t}{\cos ^{2}(t)}\right| .
\end{aligned}
$$

On the one hand, given $\varepsilon>0$ clearly there exists $\delta_{0}>0$ such that if $x<\delta_{0}$ then

$$
\int_{\alpha_{1}(x)}^{\frac{\pi}{4}} \frac{\mathrm{d} t}{\cos ^{2} t}<\varepsilon .
$$

On the other hand, using Lemma 5 for

$f(x)=\frac{1}{\cos ^{2}(x)}, \boldsymbol{\gamma}=\left\{-\alpha_{r+1}\right\}_{r=0}^{\infty}, \quad \tilde{\boldsymbol{\beta}}=\left\{\tilde{\beta}_{r}\right\}_{r=1}^{\infty}$ such that $\tilde{\beta}_{k} \in\left[\gamma_{k-1}, \gamma_{k}\right], \quad \tilde{\beta}_{2 k-1}=-\beta_{2 k-1}, a=-\alpha_{1}(x)$ and $b=0$, there exists $\delta_{1}>0$ such that if $d(\gamma)<\delta_{1}$ then

$$
\left|L(x)-\frac{1}{2} \int_{0}^{\alpha_{1}(x)} \frac{\mathrm{d} x}{\cos ^{2}(x)}\right|<\frac{\varepsilon}{2} \text {. }
$$

Since $\arctan (\cdot)$ is a continuous map on $\left(0, \frac{\pi}{4}\right)$, for $\delta_{1}$ by Lemma 6 there exists $\delta_{2}>0$ such that if ${ }_{X}<\delta_{2}$ then $d(\alpha)<\delta_{1}$.

Therefore, taking $x<\delta=\min \left\{\delta_{0}, \delta_{2}\right\}$, and replacing (15) and (16) in (14) we obtain

$$
\left|L(x)-\frac{1}{2}\right|<\varepsilon
$$

finishing the proof.

Proof of Theorem 2. The aim of the proof is to show that the limit of the three first nonzero coefficients of the power series representations of

$$
\left(\sum_{k \in \mathbb{Z}} e^{\frac{-k^{2}}{n \tau^{2}}} \operatorname{sinc}(\tau t-k)\right)^{n}
$$

and $\mathrm{e}^{-t^{2}}$ are equal for every $t \in \mathbb{R}$ and $\tau>0$ given. Indeed, for every $m \in \mathbb{N} \cup\{0\}$ and $n \in \mathbb{N}$ we fix the following notation

$$
B_{m}^{\tau}=\frac{(-1)^{m}(\pi \tau)^{2 m}}{(2 m+1) !}
$$

$$
\begin{aligned}
& C_{m, n}^{\tau}=\left\{\begin{array}{cc}
\frac{1}{2}, & \text { if } m=0, \\
\tau^{2 m} \sum_{k \in \mathbb{N}} \frac{(-1)^{k+1}}{k^{2 m}} \mathrm{e}^{\frac{-k^{2}}{n \tau^{2}}}, & \text { if } m \geq 1 ;
\end{array}\right. \\
& D_{m, n}^{\tau}=\sum_{p=0}^{m} B_{p}^{\tau} C_{m-p, n}^{\tau} .
\end{aligned}
$$

Let

$$
g(t, n)=\sum_{k \in \mathbb{Z}} \mathrm{e}^{\frac{-k^{2}}{n \tau^{2}}} \operatorname{sinc}(\tau t-k) .
$$

Note that by the analitycity is enough to consider pointwise convergence for all $t \in\left(0, \frac{1}{\tau}\right)$. Now, using expressions (17), (18), (19) and the power series of the sine function, the map $g(t, n)$ can be written in the form

$$
\begin{aligned}
& g(t, n)=\operatorname{sinc}(\tau t)+\frac{2 \tau t \sin \pi \tau t}{\pi} \sum_{k \in \mathbb{N}} \frac{(-1)^{k}}{\tau^{2} t^{2}-k^{2}} \mathrm{e}^{\frac{-k^{2}}{n \tau^{2}}} \\
& =\frac{\sin \pi \tau t}{\pi}\left(\frac{1}{\tau t}+2 \tau t \sum_{m=0}^{\infty} t^{2 m}\left(\sum_{k \in \mathbb{N}} \frac{(-1)^{k+1} \tau^{2 m}}{k^{2(m+1)}} \mathrm{e}^{\frac{-k^{2}}{n \tau^{2}}}\right)\right) \\
& =\frac{2 t}{\pi \tau}\left(\sum_{m=0}^{\infty} \frac{(-1)^{m}}{(2 m+1) !}(\pi \tau t)^{2 m+1}\right)\left(\sum_{m=-1}^{\infty} C_{m+1, n}^{\tau} t^{2 m}\right) \\
& =2\left(\sum_{m=0}^{\infty} B_{m}^{\tau} t^{2 m}\right)\left(\sum_{m=0}^{\infty} C_{m, n}^{\tau} t^{2 m}\right)=2 \sum_{m=0}^{\infty} D_{m, n}^{\tau} t^{2 m} .
\end{aligned}
$$

and therefore

$$
(g(t, n))^{n}=2^{n}\left(\sum_{m=0}^{\infty} D_{m, n}^{\tau} t^{2 m}\right)^{n}=2^{n} \sum_{m=0}^{\infty} E_{m, n}^{\tau} t^{2 m} .
$$

For $m=0$ it is clear that

$E_{0, n}^{\tau}=\left(D_{0, n}^{\tau}\right)^{n}=\left(B_{0}^{\tau} C_{0, n}^{\tau}\right)^{n}=\frac{1}{2^{n}}$ and hence 
For $m=1$ is

$$
\lim _{n \rightarrow \infty} 2^{n} E_{0, n}^{\tau}=1
$$

$$
\begin{aligned}
E_{1, n}^{\tau} & =n\left(D_{0, n}^{\tau}\right)^{n-1} D_{1, n}^{\tau} \\
& =n\left(B_{0}^{\tau} C_{0, n}^{\tau}\right)^{n-1}\left(B_{0}^{\tau} C_{1, n}^{\tau}+B_{1}^{\tau} C_{0, n}^{\tau}\right) \\
& =\frac{n}{2^{n-1}}\left(C_{1, n}^{\tau}+\frac{B_{1}^{\tau}}{2}\right) \\
& =\frac{n \tau^{2}}{2^{n-1}}\left(\sum_{k \in \mathbb{N}} \frac{(-1)^{k+1}}{k^{2}} \mathrm{e}^{\frac{-k^{2}}{n \tau^{2}}}-\frac{\pi^{2}}{12}\right) .
\end{aligned}
$$

So, using $\sum_{k=1}^{\infty} \frac{(-1)^{k+1}}{k^{2}}=\frac{\pi^{2}}{12}$,

$$
2^{n} E_{1, n}^{\tau}=-2 \sum_{k \in \mathbb{N}}(-1)^{k+1} \frac{1-\mathrm{e}^{-k^{2} n \tau^{2}}}{k^{2} n \tau^{2}}=-2 L\left(\frac{1}{n \tau^{2}}\right)
$$

where $L(\cdot)$ is introduced in Proposition 7 and now by such result we obtain

$$
\lim _{n \rightarrow \infty} 2^{n} E_{1, n}^{\tau}=-1 .
$$

For $m=2$ it follows that

$$
\begin{aligned}
E_{2, n}^{\tau}= & n\left(D_{0, n}^{\tau}\right)^{n-1} D_{2, n}^{\tau}+\frac{n(n-1)}{2}\left(D_{0, n}^{\tau}\right)^{n-2}\left(D_{1, n}^{\tau}\right)^{2} \\
= & n\left(B_{0}^{\tau} C_{0, n}^{\tau}\right)^{n-1}\left(B_{2}^{\tau} C_{0, n}^{\tau}+B_{1}^{\tau} C_{1, n}^{\tau}+B_{0}^{\tau} C_{2, n}^{\tau}\right) \\
& +\frac{n(n-1)}{2}\left(B_{0}^{\tau} C_{0, n}^{\tau}\right)^{n-2}\left(B_{0}^{\tau} C_{1, n}^{\tau}+B_{1}^{\tau} C_{0, n}^{\tau}\right)^{2} \\
= & \frac{n}{2^{n-1}}\left(\frac{B_{2}^{\tau}}{2}+B_{1}^{\tau} C_{1, n}^{\tau}+C_{2, n}^{\tau}\right) \\
& +\frac{n(n-1)}{2^{n-1}}\left(C_{1, n}^{\tau}+\frac{B_{1}^{\tau}}{2}\right)^{2}
\end{aligned}
$$

Therefore

$$
2^{n} E_{2, n}^{\tau}=F_{n}^{\tau}+G_{n}^{\tau}
$$

where

$$
\begin{aligned}
& F_{n}^{\tau}=2 n\left(\frac{B_{2}^{\tau}}{2}+B_{1}^{\tau} C_{1, n}^{\tau}+C_{2, n}^{\tau}\right) \\
& G_{n}^{\tau}=2 n(n-1)\left(C_{1, n}^{\tau}+\frac{B_{1}^{\tau}}{2}\right)^{2} .
\end{aligned}
$$

We will take the limit in each part separately. Since

$$
G_{n}^{\tau}=\frac{n-1}{2 n}\left(2^{n} E_{1, n}^{\tau}\right)^{2}
$$

from (20) we obtain

$$
\lim _{n \rightarrow \infty} G_{n}^{\tau}=12
$$

To determine the limit of $F_{n}^{\tau}$, replacing each $B_{j}^{\tau}$ and $C_{j, n}^{\tau}$ by (17) and (18), we get

$$
\begin{aligned}
F_{n}^{\tau}= & 2 n \tau^{4} \pi^{4}\left(\frac{1}{2.5 !}-\frac{1}{3 ! \pi^{2}} \sum_{k \in \mathbb{N}} \frac{(-1)^{k+1}}{k^{2}} \mathrm{e}^{\frac{-k^{2}}{n \tau^{2}}}\right. \\
& \left.+\frac{1}{\pi^{4}} \sum_{k \in \mathbb{N}} \frac{(-1)^{k+1}}{k^{4}} \mathrm{e}^{\frac{-k^{2}}{n \tau^{2}}}\right) \\
= & 2 n \tau^{4} \pi^{4}\left(\frac{1}{2.5 !}+\frac{1}{3 ! \pi^{2}} \sum_{k \in \mathbb{N}}(-1)^{k+1} \frac{1-\mathrm{e}^{-k^{2} n \tau^{2}}}{k^{2}}\right. \\
& \left.+\frac{1}{\pi^{4}} \sum_{k \in \mathbb{N}}(-1)^{k+1} k^{4} \mathrm{e}^{\frac{-k^{2}}{n \tau^{2}}}-\frac{1}{3 ! \pi^{2}} \sum_{k=1}^{\infty} \frac{(-1)^{k+1}}{k^{2}}\right)
\end{aligned}
$$

Using again $\sum_{k=1}^{\infty} \frac{(-1)^{k+1}}{k^{2}}=\frac{\pi^{2}}{12}$ and applying $\sum_{k=1}^{\infty} \frac{(-1)^{k+1}}{k^{4}}=\frac{7 \pi^{4}}{720}$, the above expression becomes

$$
F_{n}^{\tau}=2 n \tau^{4} \pi^{4}\left(\frac{-7}{720}+\frac{1}{3 ! \pi^{2}} \sum_{k \in \mathbb{N}}(-1)^{k+1} 1-\mathrm{e}^{\frac{-k^{2}}{n \tau^{2}}}\right.
$$

$$
\begin{gathered}
\left.+\frac{1}{\pi^{4}} \sum_{k \in \mathbb{N}} \frac{(-1)^{k+1}}{k^{4}} \mathrm{e}^{\frac{-k^{2}}{n \tau^{2}}}\right) \\
=2 n \tau^{4} \pi^{4}\left(\frac{1}{3 ! \pi^{2}} \sum_{k \in \mathbb{N}}(-1)^{k+1} 1-\frac{\mathrm{e}^{\frac{-k^{2}}{n \tau^{2}}}}{k^{2}}\right. \\
\left.-\frac{1}{\pi^{4}} \sum_{k \in \mathbb{N}}(-1)^{k+1} \frac{1-\mathrm{e}^{\frac{-k^{2}}{n \tau^{2}}}}{k^{4}}\right)
\end{gathered}
$$$$
=\frac{\tau^{2} \pi^{2}}{3} \sum_{k \in \mathbb{N}}(-1)^{k+1} \frac{1-\mathrm{e}^{\frac{-k^{2}}{n \tau^{2}}}}{k^{2} n \tau^{2}}-2 \tau^{4} \sum_{k \in \mathbb{N}}(-1)^{k+1} \frac{1-\mathrm{e}^{\frac{-k^{2}}{n \tau^{2}}}}{\frac{k^{4}}{n}}
$$

$$
=\frac{\tau^{2} \pi^{2}}{3} L\left(\frac{1}{n \tau^{2}}\right)-2 \tau^{2} \sum_{k \in \mathbb{N}}(-1)^{k+1} \frac{1-\mathrm{e}^{\frac{-k^{2}}{n \tau^{2}}}}{\frac{k^{4}}{n \tau^{2}}} .
$$

Therefore, since

$$
\begin{aligned}
& \lim _{n \rightarrow \infty} \sum_{k \in \mathbb{N}}(-1)^{k+1} \frac{1-\mathrm{e}^{\frac{-k^{2}}{n \tau^{2}}}}{\frac{k^{4}}{n \tau^{2}}}, \\
& =\sum_{k \in \mathbb{N}} \frac{(-1)^{k+1}}{k^{2}}=\frac{\pi^{2}}{12}
\end{aligned}
$$


using Proposition 7 is

$$
\lim _{n \rightarrow \infty} F_{n}^{\tau}=\frac{\tau^{2} \pi^{2}}{3} \cdot \frac{1}{2}-2 \tau^{2} \cdot \frac{\pi^{2}}{12}=0 .
$$

So, from here and (22), taking limits in (21) we get

$$
\lim _{n \rightarrow \infty} 2^{n} E_{2, n}^{\tau}=0+\frac{1}{2}=\frac{1}{2} \text {. }
$$

Note that from the results obtained for $m=0,1,2$ is stated that the limit of the three first nonzero coefficients of the power series representations of

$$
\left(\sum_{k \in \mathbb{Z}} \mathrm{e}^{\frac{-k^{2}}{n \tau^{2}}} \operatorname{sinc}(\tau t-k)\right)^{n}
$$

are equal to $\frac{(-1)^{m}}{m !}$, coefficients of the power series representation of $\mathrm{e}^{-t^{2}}$, ending the proof.

\section{Conclusions}

By stating property $\mathcal{P}$ a new method for computing reconstructions of a given non--band limited signal is presented. The formula is closed and stable from a numerical point view which allow to implement and do empirical experiment for other kind of signals. Our feeling is that with this approach a new frame to compute approximations of signal is opened.

\section{Acknowlegments}

The authors want to thank to Professors J. Garay and R.G. Cataln for their value comments formulated in a constructive spirit.

\section{References}

[1] L. Agud and R. G. Cataln, "New Shannon's Sampling
Recomposition," Revista de la Academia de Ciencias Zaragoza, Vol. 56, 2001, pp. 45-48.

[2] P. L. Butzer, S. Ries and R. L. Stens, "Approximation of Continuous and Discontinuous Functions by Generalized Sampling Series," Journal of Approximation Theory, Vol. 50 , No. 1, 1987, pp. 25-39. doi:10.1016/0021-9045(87)90063-3

[3] P. L. Butzer and R. L. Stens, "Sampling Theory for not Necessarily Band-Limited Functions: A Historical Overview," SIAM Review, Vol. 34, No. 1, 1992, pp. 40-53. doi: $10.1137 / 1034002$

[4] J. A. Gubner, "A New Series for Approximating Voight Functions," Journal of Physics A: Mathematical and General, Vol. 27, No. 19, 1994, pp. L745-L749. doi:10.1016/0021-9045(87)90063-3

[5] J. R. Higgings, "Five Short Stories about the Cardinal Series," Bulletin of the American Mathematical Society, Vol. 12, 1985, pp.45-89. doi:10.1090/S0273-0979-1985-15293-0

[6] H. J. Landau and H. O Pollak, "Prolate Spheroidal Wave Functions, Fourier analysis and uncertainly," Bell System Technical Journal, Vol. 40, No. 1, 1961, pp. 65-84.

[7] F. Marvasti and A. K. Jain, "Zero Crossing Bandwidth Compression, and Restoration of Nonlinearly Distorted Band-Limited Signals," Journal of the Optical Society of America, Vol. 3, No. 5, 1986, pp. 651-654. doi:10.1364/JOSAA.3.000651

[8] D. Middleton, "An Introduction to Statistical Communication Theory," McGraw-Hill, New York, 1960.

[9] C.E. Shannon, "Communication in the Presence of Noise," Proceedings of the Institute of Radio Engineers, Vol. 137, 1949, pp. 10-21.

[10] E. T. Whittaker, "On the Functions which are Represented by the Expansions of the Interpolation Theory," Proceedings of the Royal Society, Vol. 35, 1915, pp. 181-194.

[11] A. I. Zayed, "Advances in Shannon's Sampling Theory," Ed. CRC Press, Florida, 1993. 